\title{
Study of heat pipe in motor cooling: A review
}

\author{
Chen Zutao ${ }^{1, \text { a }}$, Yu Zhongjun ${ }^{1, b^{*}}, \mathrm{Fu} \mathrm{Jia}^{1}$, and Liu Bin ${ }^{1}$ \\ ${ }^{1}$ National Key Laboratory of Science and Technology on Vessel Integrated Power System, Naval Univ. of Engineering, Wuhan, 430033, \\ China
}

\begin{abstract}
The heat pipe as one of the most efficient heat exchanger device is used in many thermal engineering applications. Through sufficient literature research and summary, a comprehensive and systematic analysis of the application of heat pipe cooling technology in motor cooling is provided. The basic principles and key technologies of heat pipe cooling technology is introduced. What's more, various factors affecting the cooling efficiency of heat pipes and two main types of heat pipe applications in motor cooling are discussed. Finally, the current status of research on heat pipe cooling motors at home and abroad are reviewed.
\end{abstract}

\section{Introduction}

Heat pipe is a device that relies on fluid phase change to realize heat transfer in an enclosed body. Since its introduction in 1964, it has been widely used in defence industry, agriculture, medical and other aspects with many advantages such as good thermal conductivity, simple structure and reliable operation ${ }^{1}$, and is known as a superconductor of heat.

The application of heat pipe cooling technology in the cooling of electric motors was proposed earlier but not many practical research applications. As early as 1981, Shinichi Morita ${ }^{2}$ pointed out in the literature, heat pipes can be arranged in the motor stator outward heat conduction, or arranged in the rotor as a rotating heat pipe, and put forward specific examples of applications. However, due to the limitation of processing and manufacturing level, the application of heat pipe in motor cooling system has not been popular. With the development of science and technology, the requirements of compact structure, high power density and high reliability are put forward for the permanent magnet synchronous motor, which poses new challenges to the thermal design of the motor. The heat pipe cooling technology with simple structure, high thermal conductivity and high reliability has great advantages for solving the problems of large heat generation and concentrated heat dissipation area of new motors. Therefore, it is of great application value and strategic significance to carry out research on the application of heat pipe in motor cooling system.

Based on the full research and analysis of the application research of heat pipe in motor cooling, this paper summarizes the heat pipe cooling technology systematically, then the key issues affecting the cooling efficiency of heat pipe is analysed. Finally, focused on various applications of heat pipe in motor cooling system, an outlook on the development prospect of heat pipe technology in motor cooling application is provided.

\section{Overview of heat pipe cooling technology}

A heat pipe is a component with small cross-section, high heat transfers and high efficiency, which acts as an excellent heat conductor and is capable of transferring heat from a heat source to an external fluid environment with almost no temperature difference. As the most efficient heat transfer element, heat pipe has many applications in cooling and heat dissipation, waste heat recovery, etc., which has become one of the major energy saving and emission reduction tools utilized today.

\subsection{Principle of heat pipe cooling technology}

A heat pipe is a highly efficient heat transfer element that relies on the evaporation and condensation of a working mass inside a fully enclosed vacuum tube. Depending on the source of driving force for condensate return inside the heat pipe, heat pipes can be distinguished into rotating and non-rotating heat pipes. The non-rotating heat pipe is driven by capillary force or gravity ${ }^{4}$, while the condensate return in a rotating heat pipe is achieved by the rotational motion of the heat pipe body ${ }^{3}$.

The non-rotating heat pipe is composed of three parts: the tube shell, the suction core and the end cap. Its working principle diagram is shown in Figure $1^{4}$, which can be divided into three regions in the axial direction: the evaporation section, the evaporation transport section (adiabatic section) and the exothermic section (condensation section).

When the heat pipe is working normally, the liquid mass in the evaporation section absorbs heat and the temperature rises continuously until it reaches the phase 
change point and turns into steam, which flows to the condensation section under a small pressure difference and condenses into liquid mass after releasing heat. The liquid mass in the condensation section flows back to the evaporation section under the action of capillary driving force generated by the capillary core or its own gravity, and starts a new cycle. In this way, the heat is efficiently transferred from the evaporation section to the condensation section ${ }^{5}$.

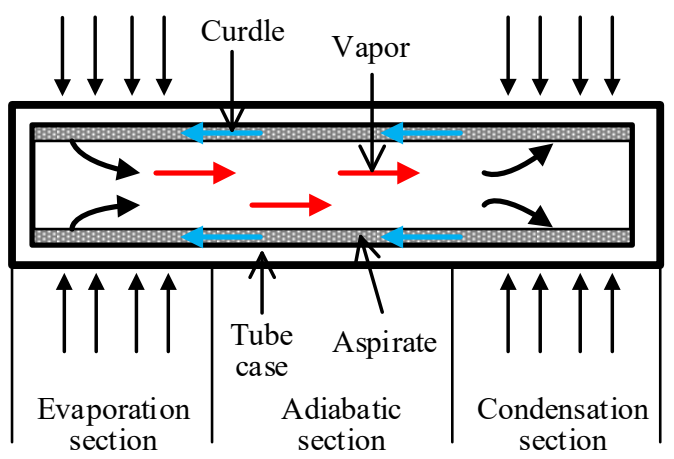

Fig. 1. Schematic diagram of capillary core heat pipe principle.

The biggest difference between rotary heat pipe and capillary core heat pipe and gravity heat pipe is that it relies on the centrifugal force of heat pipe rotation to drive the condensate reflux. A typical rotating heat pipe working principle diagram is shown in Fig. 2. The axial rotating heat pipe, for example, has a simple structure with a cylindrical appearance and no capillary core structure inside the tube ${ }^{6}$. The liquid mass is evaporated by heat absorption in the evaporation section, and the gas phase mass flows to the condensation section for exothermic condensation under the action of axial pressure difference, and so on, realizing the efficient heat transfer along the axial direction through the gas-liquid two-phase cycle.

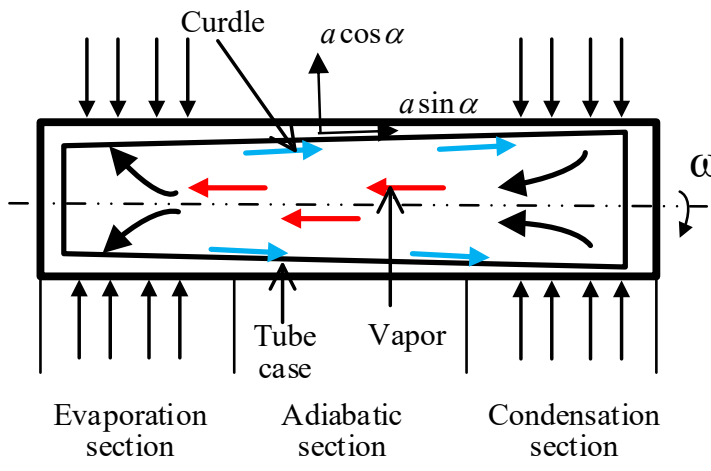

Fig. 2. Schematic diagram of rotating heat pipe principle.

\subsection{Key issues of heat pipe technology}

\subsubsection{Performance characteristics of heat pipes}

Heat pipe is a heat transfer element that relies on the phase change of its own internal mass to achieve heat transfer. It has high thermal conductivity and also has some characteristics that other heat transfer elements cannot match.
Very high thermal conductivity. Heat pipe mainly relies on the internal working liquid phase change heat transfer, and its thermal resistance is very small, which thermal conductivity can reach the good conductor copper and silver several to tens of thousands of times ${ }^{32}$, and has a very high thermal conductivity.

Heat flow direction reversibility ${ }^{26}$. The internal work of the heat pipe has spontaneous, its internal circulation power is the capillary force of the core, no external power, either end of the heat is the evaporation section, outward heat dissipation is the condensation section.

Good switching performance. Heat pipe is the use of a small temperature difference to achieve the heat transfer principle of phase change $\mathrm{e}^{13}$. It can be used to make a thermal switch or thermal diode, which only allow the heat to transfer in one direction. Thermal switch is the use of thermodynamic propagation principle. The heat transfer occurs when the heat source temperature is above a certain temperature.

Excellent environmental adaptability. The shape of the heat pipe can be changed with the conditions of the heat and cold sources. The heat pipe can be made into the rotating shaft of a motor, the blade of a gas turbine, etc. The heat pipe can also be made into a separated type to adapt to the heat transfer in the case of long distance or hot and cold fluids cannot be mixed, and the heat pipe can work normally in both gravity and gravity-free fields, so the application range is very wide $^{30}$.

\subsubsection{Factors that affect the heat transfer performance of heat pipes}

Heat pipes have a high thermal conductivity, but there are certain heat transfer limits, and their thermal conductivity is influenced by many factors.

Geometric structure parameters. The maximum heat flow rate of the heat pipe is directly determined by the geometric structure parameters. In addition to some detailed factors, such as the structure of the internal suction core of the heat pipe. Heat pipes can be divided into grooved pipe, mesh pipe and powder wick pipe, as shown in Figure 3, of which the sintered powder and combination type has a stronger thermal conductivity. For the rotating heat pipe, the moderate increase of its internal cone angle is conducive to increasing the centrifugal force of the return flow in the condensing section, which enhances the thermal conductivity ${ }^{7}$.

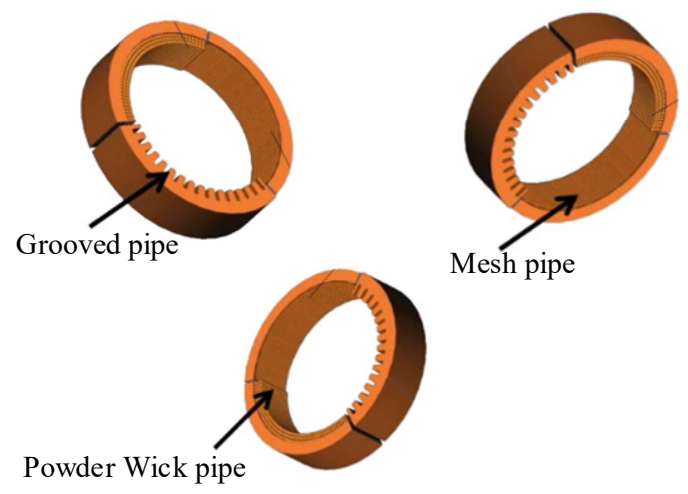

Fig. 3. Schematic diagram of tube core structure ${ }^{19}$. 
Physical parameters of the work material and fluid charge of the workmanship. The heat pipe relies on the continuous evaporation and condensation of the internal mass to achieve efficient heat transfer. The physical properties of the mass are related to many factors such as liquid density, surface tension coefficient, latent heat of vaporization, and dynamic viscosity, etc. ${ }^{8}$, which can be combined in the formula (1), which is the transmission factor, and the larger the transmission factor, the better the heat transfer performance.

$$
N=\frac{\sigma \cdot \rho_{l} \cdot h_{f g}}{\mu_{l}}
$$

The amount of liquid filling also has a greater impact on the heat pipe start and transfer performance. If the amount of work material filling is too little, it will lead to the phenomenon of steam dry, and eventually make the heat pipe operation failure; if the amount of work material filling is too much, it is easy to form a liquid plug, so that it can not work properly, affecting the overall performance.

The impact of working conditions. The performance of the heat pipe is also affected by the working environment. For capillary core heat pipe and gravity heat pipe, the size of the placement angle will influence the heat pipe to start or work. The rotating heat pipe is also affected by the speed of the rotor. All of these factors will affect the heat pipe thermal conductivity ${ }^{27}$.

\section{The research status of heat pipe in motor cooling}

With the increase in cooling system requirements for integrated, high power density motors, a lot of research has been conducted at home and abroad on the use of heat pipe technology for motor cooling.

Heat pipe for motor cooling is mainly divided into two forms: one is installed in the heat-generating parts of the stator and rotor of the motor, exporting heat to the two ends of the motor where it is easy to dissipate, thus reducing the temperature rise of the motor. The other is the rotating heat pipe motor, which make the rotor itself into a rotating heat pipe, the heat of the motor rotor is brought to one end of the motor through the rotating heat pipe to dissipate, and at the same time the temperature rise of the stator winding is also reduced. The use of both forms of heat pipes can increase the power of the motor while maintaining the winding insulation and heat resistance requirements.

\subsection{Stator and rotor heat pipe cooled motors}

Motors with heat pipe cooling for stator and rotor generally have two structures: gravity heat pipe cooling and normal heat pipe cooling. Several typical heat pipes used for motor stator and rotor cooling are shown in Table 1. Gravity heat pipes are mainly arranged along the motor axial direction to cool the stator coil and stator core respectively, and ordinary shaped heat pipes are used by distributing several heat pipes evenly along the circumference in the motor casing, stator slot bottom or stator yoke part. In order to strengthen the cooling effect of the cooling section, there are more cooling fins in the condensing section.

Schneider ${ }^{9}$ et al, in 1994, proposed a thermal siphon tube cooling device for permanent magnet motors with electric actuators, where gravity heat pipes are arranged along the radial direction in the motor housing to achieve cooling of the motor, ensuring that the motor temperature does not exceed the limit temperature and achieving a motor temperature drop up to $90 \mathrm{~K}$. In 2016, Xiaozhi Qiu ${ }^{10}$ et al. designed a loop heat pipe cooling system for airborne electromechanical actuators with permanent magnet synchronous motors that can better adapt to the working conditions of the electromechanical actuator with fully enclosed structure and no internal ventilation, and achieves a better cooling effect. The cooling system uses a flat plate evaporator with water as the cooling medium, which can reduce the motor temperature from $337^{\circ} \mathrm{C}$ to $147^{\circ} \mathrm{C}$ under natural cooling conditions, achieving high cooling efficiency. 2020, Wenjing Niu ${ }^{11}$ et al. designed a heat pipe-fuel based cooling system for airborne drive motors. The stator was cooled using a heat pipe placed in the groove of the outer wall of the motor stator, and the fuel oil served as the condenser of the heat pipe to absorb heat from the heat pipe. The structure arranges the heat pipe along the axial direction, which also avoids the loss brought by the heat pipe under the condition of satisfying the cooling effect small, and at the same time, in order to reduce the mass carried by the cooling system, aircraft fuel is used as the cooling medium to realize the cooling of the heat pipe, which can better improve the energy density of the system and also achieve a good cooling effect. In 2017, South China University of Technology ${ }^{12}$ proposed a new type of inner rotor permanent magnet motor for electric vehicles water-cooled, heat-tube composite cooling scheme for motor cooling. The heat pipe and motor were determined to improve the heat dissipation efficiency of the motor end windings by following a connection method that connects one end of the heat pipe to the end cover and the other end extends into the motor interior and combines with the end windings using electronic potting glue. Two heat pipe based motor heat dissipation configurations are discussed: tail straight tube and tail bend type. The tail straight heat pipe arrangement is slightly less effective, which can reduce the maximum temperature rise of the winding by about $14.8^{\circ} \mathrm{C}$. Harbin Institute of Technology ${ }^{13}$ conducted a study on the cooling system of high power density permanent magnet motor for high altitude vehicle, which uses heat pipe axial cooling to improve the cooling efficiency of the cooling system. The temperature distribution of the motor under the three cases of no heat pipe cooling, heat pipe enhanced cooling, and heat pipe assisted plus air cooling were discussed respectively. The results show that the heat pipe achieves a very good effect on the control of motor temperature rise, which can reduce the maximum motor temperature rise by $131^{\circ} \mathrm{C}$ and has a high cooling efficiency.

In 2017 the University of Nuremberg in Erlangen ${ }^{14}$ used flat heat pipes instead of conventional water jackets to implement cooling of stators and experimentally evaluated this cooling method. It is shown that the application of heat pipes on the stator surface avoids the 
problem of axial temperature rise of the coolant in the conventional water jacket cooling system. At the same time, the heat pipe acts as a carrier of heat dissipation from the stator surface, simplifying the cooling system and reducing costs. It was demonstrated that this cooling method can be more easily applied to existing motors to improve machine efficiency or increase machine power. In 2019, the Clemson University ${ }^{15}$ investigated a hybrid cooling system that combines heat pipes with conventional liquid cooling in a compact thermal support. The design allows heat to be dissipated through an integrated thermal path by regulating various actuators (e.g., centrifugal fans, radiator pumps, and fans) to minimize energy consumption. The results show that the motor temperature is maintained at a target value of approximately $70^{\circ} \mathrm{C}$. In addition, this hybrid solution offers significant energy consumption advantages compared to conventional liquid cooling systems. In 2018, the University of Edinburgh ${ }^{16}$ investigated the effect of the use of natural air cooling, direct liquid cooling and heat pipes on motor temperature, developing a winding coil with heat pipes and discussing the cooling effect of different heat pipe shapes and locations. The experimental results showed that the L-shaped heat pipe located at the outer diameter of the winding dissipated the heat best and reduced the hot spot temperature of the winding by $20 \%$ compared to the winding without the heat pipe. The performance of the motor thermal management system using the L-shaped flat heat pipe was experimentally investigated at the University of Indonesia ${ }^{17}$ in 2017 . The prototype L-shaped heat pipe evaporative section was placed inside the motor housing and the condensing section was partially placed outside the motor housing and cooled with circulating liquid or air flow. The experiments showed that the cooling system can reduce the motor housing temperature by $33.8^{\circ} \mathrm{C}$ and has a good cooling effect.

Table 1. Several typical heat pipe cooled motors.

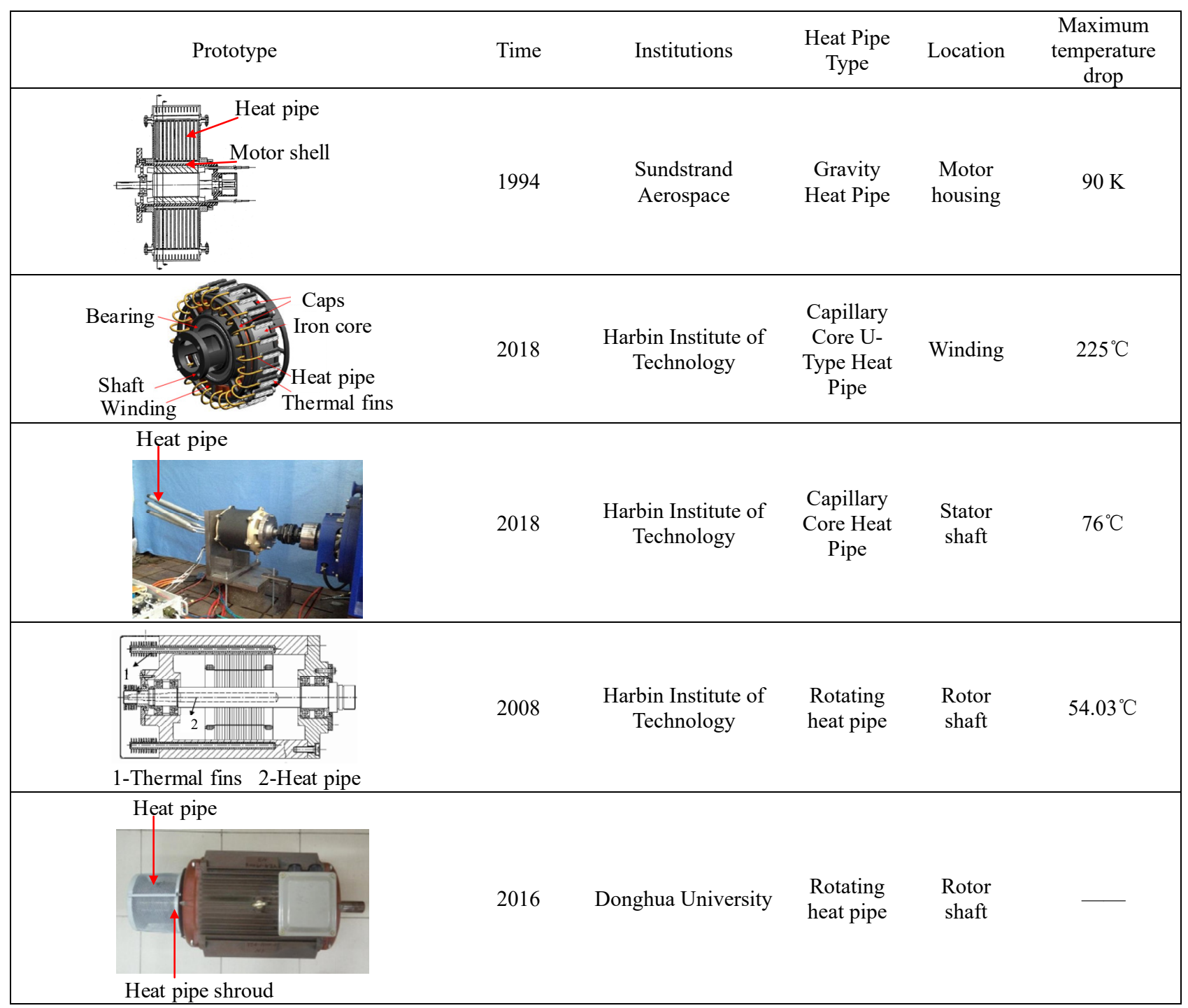




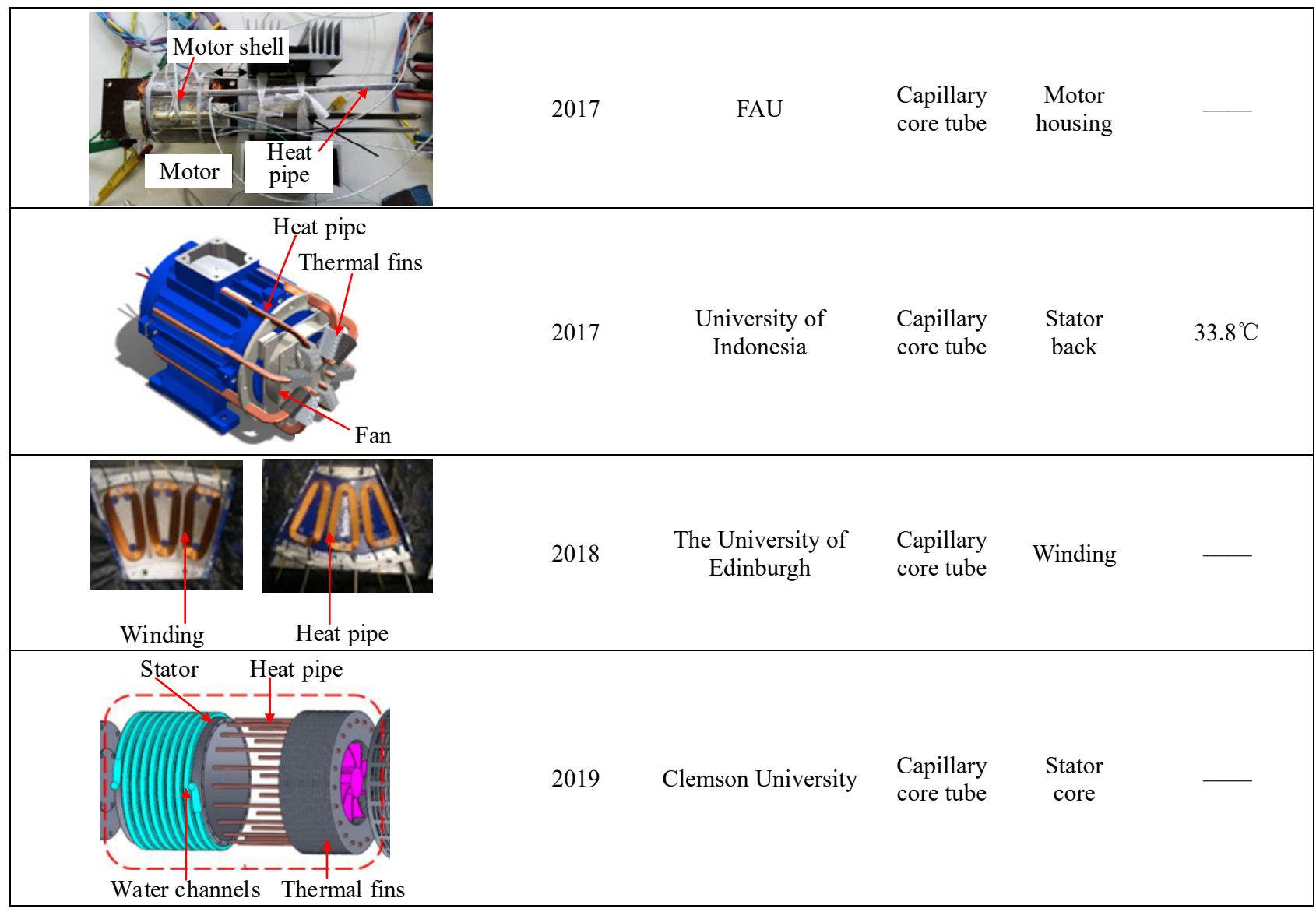

\subsection{Heat pipe shaft cooling motor}

Shaft cooling is an early and successful example of rotating heat pipes used for motor cooling. In a heat pipe shaft motor, a rotating heat pipe is inserted into the centre hole of the rotor and relies on the tight fit between the heat pipe and the shaft to absorb the heat from the rotor and vaporize the mass. The outer end of the rotor is equipped with heat sink fins, and the heat is removed by fan ventilation, so that the work vapour condenses and flows back to the heat producing section of the rotor. With the progress of processing technology and the improvement of integration, the heat pipe and the rotor are gradually adopted to make a single shaft.

In 2008, Harbin Institute of Technology ${ }^{8}$ proposed the integration of rotating heat pipe in the spindle unit to achieve cooling for the machine tool spindle motor built into the spindle unit with high speed and high heat generation. The research discussed and analysed the temperature control effect of oil cooling and heat pipe cooling on the electric spindle, and the results showed that the cooling effect of electric spindle based on heat pipe cooling is more obvious and the temperature gradient distribution of the spindle is more uniform. The simulation also analysed that forced cooling in the condensing section of the heat pipe or adding a rotating heat pipe to the spindle core can enhance the cooling of the electric spindle. In 2016, scholars from Donghua University ${ }^{20}$ designed a heat pipe installed in the rotor to conduct heat to the rotor in response to the problem of difficult heat dissipation of the motor rotor working in confined space. The study established a finite element model of the heat pipe and rotor coupling, and analysed the effects of different connection methods on contact stress and temperature diffusion. In 2020, Shenyang University of Technology ${ }^{18}$ applied rotating heat pipe technology to vacuum dry pump drive motors, and according to the structural characteristics of vacuum dry pump drive motors, the location of the end of the rotor shaft in contact with the bearing was used as the evaporation section of the heat pipe, and the heat sink was added at the bearing location to increase the condensation section. The heat dissipation of the condensing section is increased by adding a heat dissipation fan at the position of the motor of the whole unit casing to increase the heat dissipation of the evaporating section, and a very good heat dissipation effect is obtained.

\section{Conclusion}

Since the introduction of heat pipes in the 1970s, the research and development of heat pipe cooling technology has become more and more comprehensive, and with the continuous development of modern manufacturing technology and quality inspection processes, some of the problems of compatibility and reliability in the deployment of heat pipe technology in electric motors have been gradually solved. Due to the rapid development of integrated, high power motors, efficient and reliable heat pipe cooling will also be more popular in the motor cooling industry. 
This paper reviews the application of heat pipe cooling technology in motor cooling systems from the working principle of heat pipe technology, focusing on the development of the cooling technology and the current status of research at home and abroad, and the related technologies and problems are summarized. The existing heat pipe cooling motor technology can be divided into 2 categories, namely stator and rotor heat pipe cooling and rotor shaft heat pipe cooling. Stator and rotor heat pipe cooling usually adopts gravity heat pipe and capillary core heat pipe, which are arranged in the back of stator, core, winding and other parts to transfer heat, while rotor shaft heat pipe cooling usually adopts rotating heat pipe, which is directly integrated in the rotor shaft to transfer heat to both ends of the rotor shaft. The cooling performance advantages of both methods have proven to be very obvious.

This technological development will bring new efficient and integrated solutions for thermal management of motors, while the gradual emergence of highperformance heat pipes such as heat pipes with nanomaterials as filler masses and flexible heat pipes will promote the application of cooling technology in integrated motors for automotive and aerospace propulsion/on-board power generation, making heat pipe technology a more direct and feasible cooling implementation solution.

\section{References}

1. Yang Q Y, Song X R. (1989) Design and experimental analysis of rotating heat pipes for electric motors [J]. Energy Engineering, (01):10-12.

2. Morita, Shinichi, Yang, H. D., Wu, Y. M.. (1982) Application of heat pipes to electric motors [J]. Motor Technology, (3):32-35.

3. Jankowski T A. (2007) Numerical and Experimental Investigations of a Rotating Heat Pipe[R].Los Alamos National Laboratory (LANL), Los Alamos, NM.

4. SHUKLA K N. (2015) Heat pipe for aerospace applications-an overview[J]. Journal of Electronics Cooling and Thermal Control,5(1):1-14.

5. Li J W, Dai S G. (2019) Research progress and outlook of high temperature heat pipe technology[J]. China Space Science and Technology,39(03):30-42.

6. Wei J W, Yang K, Xie Y, et al. (2011) Threedimensional temperature field of a high-rotation differential step generator rotor and application of rotating heat pipe $[\mathrm{J}]$. Journal of Electrical Machines and Control, 15(7): 82-86.

7. Matt A. Streby, Pon R. (1996) Ponnappan, John E. LelandJ, Jerry E. Beam. Design and testing of a high speed rotating heat pipe. Proceedings of the intersociety energy conversion engineering conference. Sponsored IEEE, 1453 1458.

8. Bi L N. (2008) Performance of electric spindle heat pipe for machine tools and its experimental study [D]. Harbin Institute of Technology.

9. Schneider M, Bland T. (1994) Preliminary test results of reflux-cooled electromechanical actuator[J]. Aiaa Journal.

10. Qiu S. C. (2016) Research on heat dissipation of electromechanical actuator motors for multi-electric aircraft. Nanjing University of Aeronautics and Astronautics.

11. Niu W J, Lian W L, Lin L J, et al. (2020) Heat pipe technology-based heat dissipation characteristics of aircraft electric actuators[J]. Journal of Aerodynamics, v.35(04):44-54.

12. Wen W Y. (2017) Design and performance analysis of heat pipe based heat sink system for new energy vehicle motor [D]. South China University of Technology.

13. Zhang J P. (2018) Research on high efficiency and high power density permanent magnet synchronous motors for high altitude vehicles [D]. Harbin Institute of Technology.

14. Lindner A., Hahn I., (2017) Practical Evaluation of a Passive Stator Cooling Concept without Thermal Stacking, IEEE Workshop on Electrical Machines Design, Control and Diagnosis (WEMDCD), pp. 132 -139 .

15. Huang J., Nain S. S. i, R. Miller, D. Rizzo, K. Sebeck, S. Shurin, J. Wagner, (2019) A Hybrid Electric Vehicle Motor Cooling System - Design, Model, and Control, IEEE Transactions on Vehicular Technology, vol. 68 , no. 5 , pp. $4465-4478$.

16. Muelle M. A. r, Burchell J., Chong Y. C., O. Keysan, A. McDonald, M. Galbraith, E. J. P. Echenique Subiabre, (2019) Improving the Thermal Performance of Rotary and Linear Air-Cored Permanent Magnet Machines for Direct Drive Wind and Wave Energy Applications, IEEE Transactions on Energy Conversion, vol. 34, no. 2, pp. $773-781$.

17. Putra N., Ariantara B., (2017) Electric Motor Thermal Management System using L-Shaped flat Heat Pipes, Applied Thermal Engineering, Elsevier, vol. 127, pp. $1156-1163$.

18. Yang W G. (2020) Analysis of a new method and engineering implementation for rotor heat dissipation of special motors for vacuum dry pumps [D]. Shenyang University of Technology.

19. Wakefield. (2019) www.wakefield-vette.com.

20. Chen, C.S.. (2016) Research on energy-saving technology and application of thermal conductivity and heat dissipation in electric motors [D]. Donghua University.

21. He Y B. (2012) Phase change heat transfer study of liquid nitrogen heat pipe subcooler for high temperature superconducting motor [D]. Harbin Institute of Technology.

22. Gong C Y. (2012) Research on intelligent control of solar water heater based on stepper motor [D]. Nanchang University.

23. Ma, B. F.. (2008) Research on the thermal performance of liquid hydrostatic electric spindles based on heat pipe heat transfer and related 
technologies [D]. Harbin Institute of Technology. Schneider, M., \& Bland, T. (1994). Preliminary test results of reflux-cooled electromechanical actuator. Aiaa Journal.

24. Wu J N, (2019) Nuclear grade 1 stainless steel Ushaped heat transfer tube for external heat exchanger of large advanced pressurized water reactor main pump shield motor. Zhejiang Province, Zhejiang Juli Special Material Technology Co.

25. Wang Y S. (2019) Research on the heat dissipation effect of automotive motor based on heat pipe improvement[J]. Automotive Practical Technology.: 137-141.

26. Zhang Y. (2019) Design and performance study of automatic powder filling machine for copper powder sintered heat pipe[D]. South China University of Technology.

27. Sun Y L. (2019) Design and performance analysis of heat pipe type heat sink system for permanent magnet synchronous motor[D]. South China University of Technology.

28. Wang S P, WU B X, Wen W Y, He J B. (2018) Thermal analysis of new energy vehicle motor based on heat pipe - air cooling system[J]. Motor and Control Applications, 45(08):91-97+109.

29. Zhang X T. (2018) Research on high-efficiency and high-torque density permanent magnet synchronous motor for solar UAV [D]. Harbin Institute of Technology.

30. Chen, C.S., Chen, G., Liang, S.Q. (2017) Finite element analysis and optimization of heat-conducting tube and motor rotor overfilling coupling[J]. Journal of Donghua University (Natural Science Edition), 43(06):902-907.

31. Liu, T. (2017) Research and development of automatic temperature difference detection system for solar gravity heat pipe batching[D]. China University of Metrology.

32. Zhai Z K. (2017) Research on heat transfer performance of rotating heat pipe and its application exploration in anti-icing of fairing[D]. Nanjing University of Aeronautics and Astronautics.

33. Hu J C, Yue YL, Wang T H, Yuan G, Zhang ZS. (2001) Application and development status of heat pipe[J]. Refrigeration, (03):20-26.

34. Dai Q Z. (1992) The application of heat pipe in motor cooling $[\mathrm{J}]$. Journal of Electrical Engineering Technology.

35. Yang Q Y. (1987) Application of rotating heat pipe on electric motor[J]. Modern Energy Conservation.

36. Huang, W.K., Liu, C.W.. (1985) Application of rotating heat pipe on three-phase asynchronous speed control motor[J]. Small and medium-sized motors.

37. Liu R, Lin G P. (2010) Study of loop heat pipe improvement for electric actuator heat dissipation[J]. Journal of Civil Aviation Flight Academy of China, 21(1):48-51. 